\title{
Automatic Lung Surface Registration Using Selective Distance Measure in Temporal CT Scans
}

\author{
Helen Hong ${ }^{1}$, Jeongjin Lee ${ }^{2}$, Kyung Won Lee ${ }^{3}$, and Yeong Gil Shin ${ }^{2}$ \\ ${ }^{1}$ School of Electrical Engineering and Computer Science BK21: Information Technology, \\ Seoul National University, San 56-1 Shinlim 9-dong Kwanak-gu, Seoul 151-742, Korea \\ hlhong@cse.snu.ac.kr \\ ${ }^{2}$ School of Electrical Engineering and Computer Science, Seoul National University \\ \{jjlee,yshin\}@cglab.snu.ac.kr \\ ${ }^{3}$ Dept. of Radiology, Seoul National University Bundang Hospital, 300, Gumi-dong, \\ Sungnam-si, Kyunggi-do, Korea \\ lkwrad@radiol.snu.ac.kr
}

\begin{abstract}
In this paper, we propose a novel technique of lung surface registration for investigating temporal changes such as growth rates of pulmonary nodules. For the registration of a pair of CT scans, a proper geometrical transformation is found through the following steps: First, optimal cube registration is performed for the initial gross registration. Second, for allowing fast and robust convergence on the optimal value, a 3D distance map is generated by the local distance propagation. Third, the distance measure between surface boundary points is repeatedly evaluated by the selective distance measure. Experimental results show that the performance of our registration method is very promising compared with conventional methods in the aspects of its computation time and robustness.
\end{abstract}

\section{Introduction}

Chest computed tomography (CT) is a sensitive method for detecting pulmonary nodules [1]. In clinical practice, radiologists often compare current chest CT with previous one of the same patient to investigate temporal changes such as growth rates of pulmonary nodules. However, it is often very difficult even for radiologists to identify subtle changes, particularly in lesions that involve overlap with anatomic structures such as ribs, vessels, heart, and diaphragm. The automatic detection of corresponding regions in temporal CT scans would be very useful for detecting and tracing pulmonary nodules

Several methods have been suggested for the automated matching of temporal lung CT images. In Betke et al. [2-4], anatomical landmarks such as the sternum, vertebrae, and tracheal centroids are used for initial global registration. Then the initial surface alignment is refined step by step by an iterative closest point (ICP) process. However most part of the computation time for the ICP process is to find the point correspondences of lung surfaces obtained from two time interval CT scans. Hong et al. [5] proposed an efficient multilevel method for surface registration to cope with the problem of Betke [2]. The multilevel method first reduces the original number of points and aligned them using an ICP algorithm. In addition, they proposed a midpoint approach to define point correspondences instead of using the point with the smallest Euclidean distance as in the original ICP algorithm. However the midpoint 
approach has a tradeoff between accuracy and efficiency, because additional processing time is needed to find the second closest point and compute the midpoint. Mullaly et al. [6] developed a multi-criterion nodule segmentation and registration methods that facilitate the identification of corresponding nodules in temporal chest CT scans. The method requires additional nodule segmentation and measures for multi-criterion. Gurcan et al. [7] developed an automated global matching of temporal thoracic helical CT scans. The method uses three-dimensional anatomical information such as the ribs but did not require anatomical landmark identification or organ segmentation. However it is difficult to align correctly since the method uses only the reduced information obtained by Maximum Intensity Projection (MIP) images of two time-interval CT scans.

Current approach still needs some progress in computational efficiency and accuracy for investigating changes of lung nodules in temporal chest CT scans. In this paper, we propose a novel technique of lung surface registration to speed-up the computation time and increase robustness. Our proposed registration method for aligning a pair of CT images is composed of three procedures. First, optimal cube registration is performed to correct gross translational mismatch. This initial registration does not require any anatomical landmarks. Second, a 3D distance map is generated by local distance propagation, which derives fast and robust convergence on the optimum value. Third, the distance measure between surface boundary points is evaluated repeatedly by the selective distance measure. Then the final geometrical transformations are applied to align lung surfaces in the current CT scans with lung surfaces in the previous CT scans. Experimental results show that our method is dramatically faster than the chamfer matching-based registration and more robust in the sense that the algorithm always converges to an optimum value.

The organization of the paper is as follows. In Section 2, we discuss how to correct gross translational mismatch. Then we propose a local distance propagation and selective distance measure to find exact geometrical relationship in the two time interval images. In Section 3, experimental results show how the method rapidly and robustly aligns lung surfaces of current and previous CT scans. This paper is concluded with a brief discussion of the results in Section 4.

\section{Lung Surface Registration}

For the registration of the current CT study, called target volume, with the previous study, called template volume, we apply the pipeline shown in Fig. 1 to the temporal CT scans. At first, lung surfaces are automatically segmented from each volume and saved as binary volumes. In the second step, initial alignment is performed using the optimal cube registration for correcting the gross translational mismatch. In the third step, initial alignment is repeatedly refined by the selective distance measure and optimization. In order to find exact geometrical relationship between two time interval volumes, the target volume is moved during the iterative alignment procedure. Interpolating the target volume at grid positions of the template volume is required for the each iteration depending on the transformation. After registration, lung surfaces of template and target volume are displayed by data mixing and volume rendering.

In our method, we have two assumptions as follows: 1) Each CT scan is acquired at the maximal respiration. 2) The entire lung regions are included in each CT scan. Based on this assumption, in general, rigid transformation is sufficient for the regis- 
tration of temporal chest CT scans. We use rigid transformation - three translations and three rotations about the $x$-, $y$-, $z$-axis.

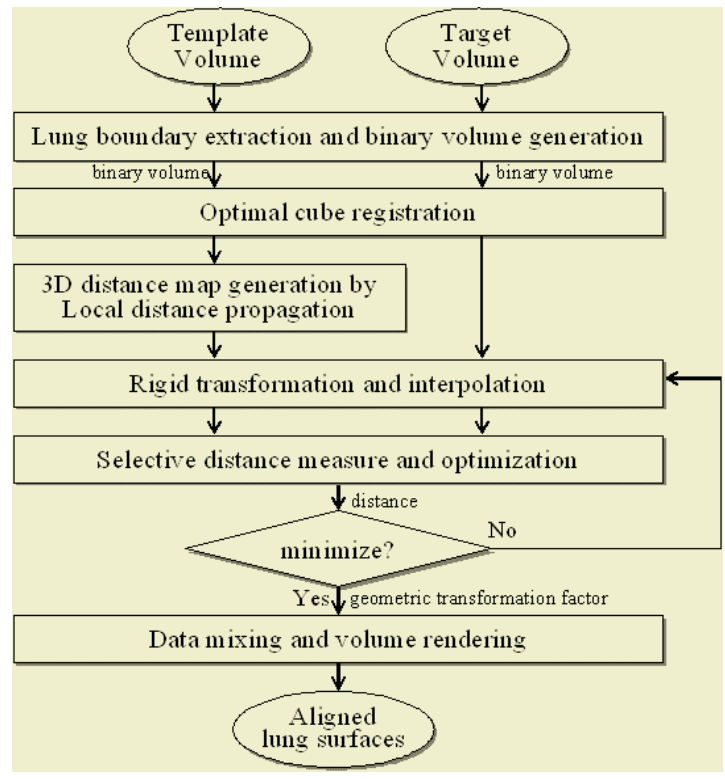

Fig. 1. The pipeline of automatic lung surface registration

\subsection{Optimal Cube Registration}

According to the imaging protocol and the patient's respiration and posture, the position of lung surfaces between template and target volume can be quite different. For the efficient registration of such volumes, an initial gross correction method is usually applied. Several landmark-based registrations have been used for the initial gross correction. To achieve the initial alignment of the lung surfaces, these landmark-based registrations require landmark detection and point-to-point registration of corresponding landmarks. These additional processes much degrade the performance of the whole system.

To minimize the time and maximize the effectiveness for initial registration, we propose a simple method of global alignment using the circumscribed boundary of lung surfaces. An optimal cube of bounding volume which includes left and right lung surfaces is generated as shown in Fig. 2. For initial registration of two volumes, we align centers of optimal cubes.

Our optimal cube registration dramatically reduces the processing time since initial alignment is performed without any anatomical landmark detection. In addition, our method leads to robust convergence to the optimal value since the search space is limited near optimal value.

\subsection{Local Distance Propagation}

In a surface registration algorithm, the calculation of distance from a surface boundary to a certain point can be done using a preprocessed distance map based on the 


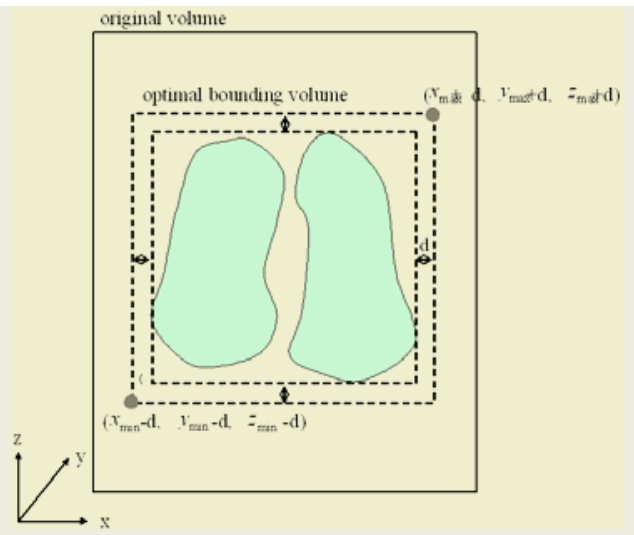

Fig. 2. The generation of an optimal cube

chamfer matching. The method reduces the generation time of a distance map by an approximate distance transformation instead of a Euclidean distance transformation. However the computation time of distance is still expensive by two-step distance transformation using forward and backward mask. In particular, the generation of a 3D distance map of whole volume dataset is unnecessary when the initial alignment almost corrects the gross translational mismatch. From this observation, we propose a local distance propagation for the efficient generation of a distance map.

For generating a 3D distance map, we approximate the global distance computation with repeated propagation of local distances within a small neighborhood. To approximate Euclidean distances, we consider 26-neighbor relations for a 1-distance propagation as seen in Eq.(1). The positive distance value of the 3D distance map tells how far it is apart from the surface boundary points. The local distance propagation shown in Fig. 3 is applied to the surface boundary points only in the template volume.

$$
D P(i)=\min \{D P(i-1)+1, D P(i-1)\}
$$

Fig. 4 shows the result of a 3D distance map using local distance propagation. The generation time is considerably reduced since pixels only need to be propagated in the direction of increasing distances to the maximum neighborhood. In addition, we do not need the backward propagation which reduces the size of neighborhoods used.

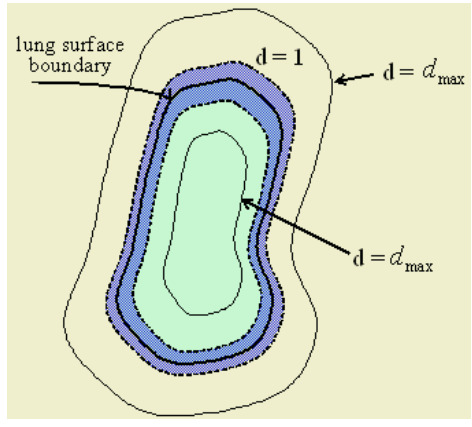

Fig. 3. Local distance propagation

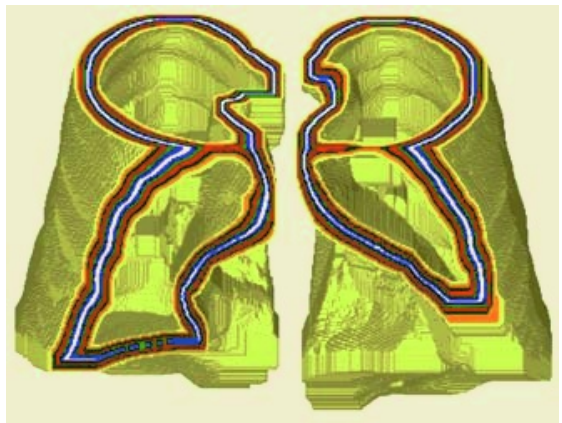

Fig. 4. The result of 3D distance map 


\subsection{Selective Distance Measure and Optimization}

The distance measure is used to determine the degree of resemblance of surface boundaries of template and target volume. To get the distance measure, the current approach needs to calculate the root mean square of distance differences of whole values in a distance map. Whereas, our selective distance measure(SDM) only uses distance values near to the surface boundary that can be found in the already generated a 3D distance map. Since distance values in the selected regions are used, we can reduce the computation time for the distance measure and optimization.

As can be seen in Eq. (2), the distance value $D_{\text {target }}(i)$ of target volume is subtracted from the distance value $D_{\text {template }}(i)$ of the 3D distance map of template volume. We assume that $D_{\text {target }}(i)$ are all set to $0 . N_{C}$ is the total number of surface boundary points in target volume. Then $S D M$ reaches minimum when surface boundary points of template and target volume are aligned correctly.

$$
S D M=\frac{1}{N_{C}} \sum_{i=0}^{N_{C}-1}\left|D_{\text {template }}(i)-D_{\text {target }}(i)\right|
$$

We use the Powell's method for evaluating SDM. Since the search space of our distance measure is limited to the surrounding lung surface boundaries, we do not need to use a more powerful optimization algorithm such as simulated annealing.

\section{Experimental Results}

All our implementation and test were performed on an Intel Pentium IV PC containing 2.4 GHz CPU and 1.0 GBytes of main memory. Our registration method has been applied to three pairs of successive CT scans whose properties are described in Table 1. The performance of our method is evaluated with the aspects of visual inspection, accuracy and robustness.

Table 1. Experimental datasets

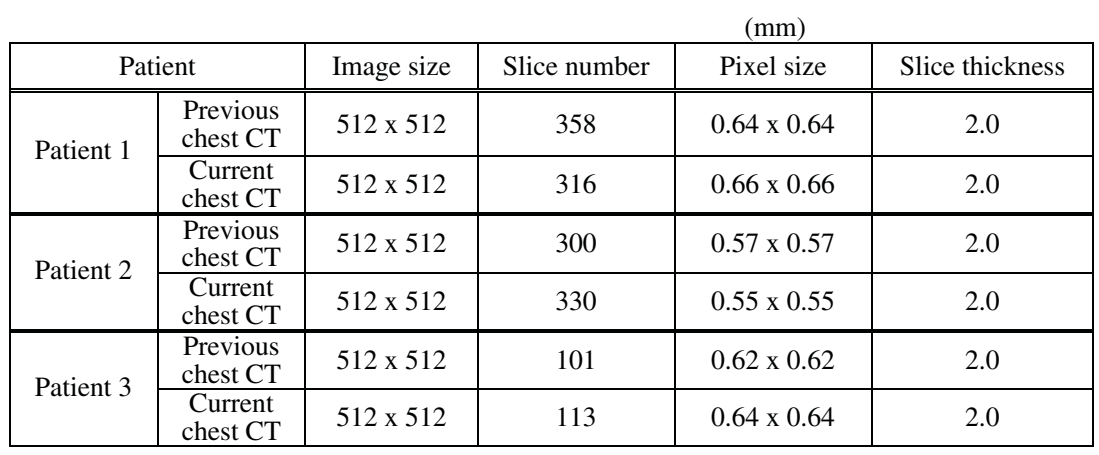

Fig. 5 shows the effectiveness of the optimal cube for initial registration. The positional difference between lung surfaces of template and target volume shown in Fig. 5 (a) are much reduced as shown in Fig. 5 (b) by the optimal cube registration. This 
initial registration is further refined until lung surfaces of template and target volume are aligned exactly like a Fig. 6 (c) and (d).

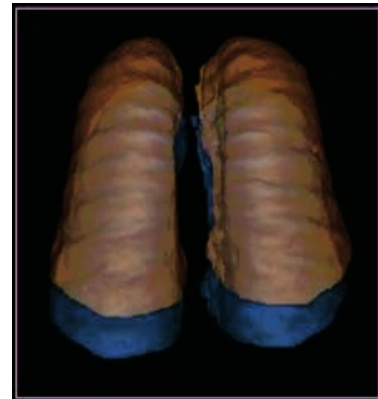

(a)

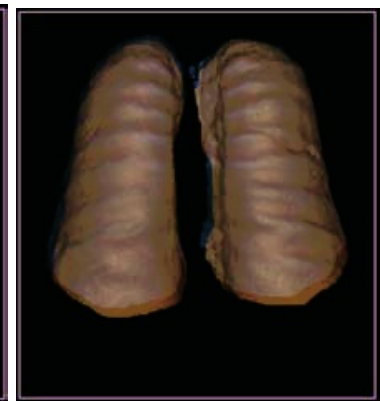

(b)

Fig. 5. The results of optimal cube registration (a) initial position (b) after initial registration

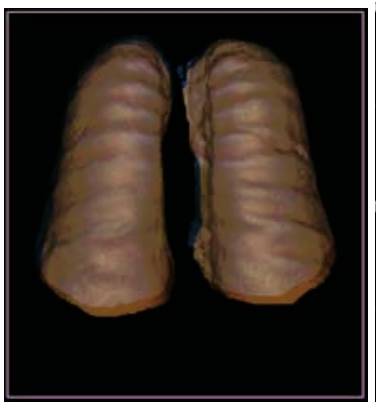

(a)

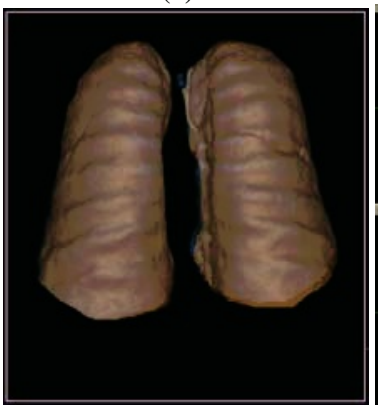

(c)

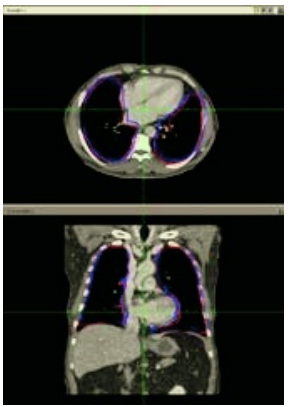

(b)

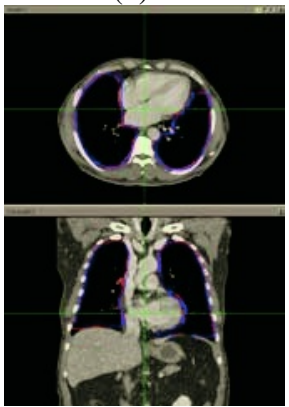

(d)

Fig. 6. The results of lung surface registration (a) after initial registration in 3D view (b) after initial registration in axial and coronal view (c) after surface registration in 3D view (d) after surface registration in axial and coronal view

Fig. 7 shows how we can reduce the registration time and the error measure, the sum of squared distance difference (SSD), using the optimal cube registration. After the first iteration, the SSD of our method is significantly reduced by optimal cube registration compared to chamfer matching-based surface registration. Moreover in the early iterations the SSD of our method (Method 2) is much smaller than that of chamfer matching-based surface registration (Method 1), as shown in Fig. 8. 


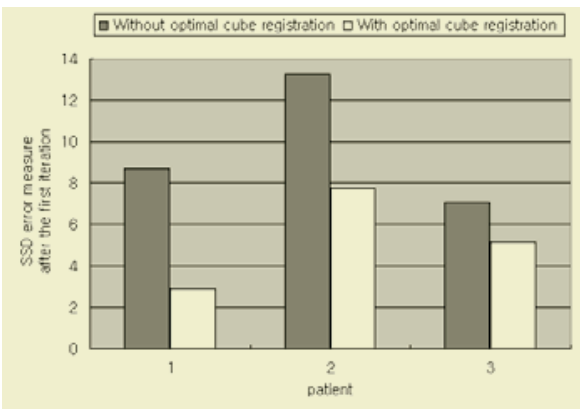

(a)

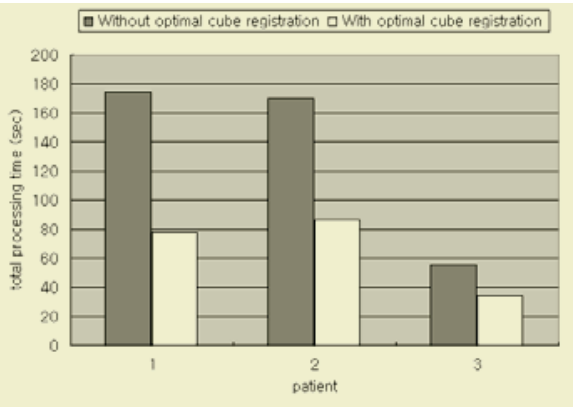

(b)

Fig. 7. The effectiveness of the optimal cube registration (a) SSD error measure (b) total processing time

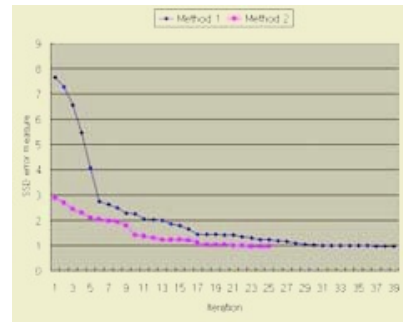

(a)

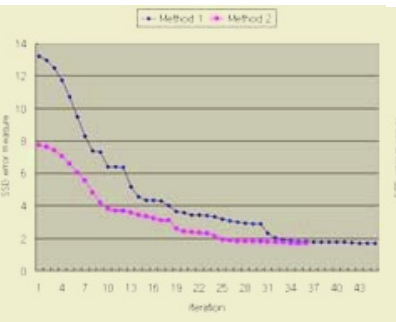

(b)

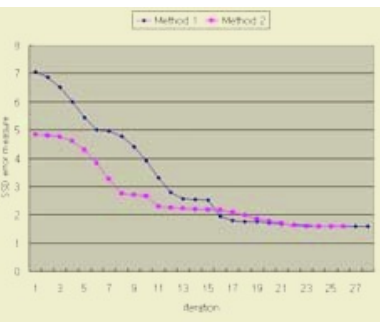

(c)

Fig. 8. Accuracy evaluation using SSD per iteration (a) patient 1 (b) patient 2 (c) patient 3

The total processing time is summarized in Table 2 where execution time is measured for the generation of a distance map, the distance measure and optimization.

The robustness of the selective distance measure (SDM) criterion has been evaluated by comparing SDM measure traces (represented by square dot line) with chamfer distance measure (CDM) traces (represented by diamond dot line). As shown in Fig. 9 , the changes of SDM measure are smooth near to the minimal position, but CDM measure is changed rapidly. This means that SDM measure is more likely to converge to an optimum value.

\section{Conclusion}

We presented a novel technique of lung surface registration of a pair of chest CT scans. Using the optimal cube registration, the initial gross registration can be done much fast and effectively without any detection of anatomical landmarks. Selective distance measure using a 3D distance map generated by the local distance propagation allows rapid and robust convergence on the optimal value. Three pairs of temporal chest CT scans have been used for the performance evaluation with the aspects of visual inspection, accuracy and robustness. In the early iterations, the SSD of our method is much smaller than that of chamfer matching-based registration by using optimal cube registration and selective distance measure. Experimental results also show that SDM measure has more chance of converging to an optimum value than CDM measure. Our method can be successfully used for investigating temporal changes such as growth rates of pulmonary nodules. 
Table 2. Total processing time

\begin{tabular}{|c|c|c|c|c|}
\hline \multicolumn{2}{|c|}{ Patient } & $\begin{array}{c}\text { 3D distance map } \\
\text { generation }\end{array}$ & $\begin{array}{c}\text { Distance measure and } \\
\text { optimization }\end{array}$ & Total processing time \\
\hline \multirow{2}{*}{ Patient 1 } & Method 1 & 46 & 120 & 166 \\
\cline { 2 - 5 } & Method 2 & 5 & 72 & 77 \\
\hline \multirow{2}{*}{ Patient 2 } & Method 1 & 41 & 122 & 163 \\
\cline { 2 - 5 } & Method 2 & 7 & 78 & 85 \\
\hline \multirow{2}{*}{ Patient 3 } & Method 1 & 11 & 43 & 54 \\
\cline { 2 - 5 } & Method 2 & 4 & 34 & 38 \\
\hline
\end{tabular}

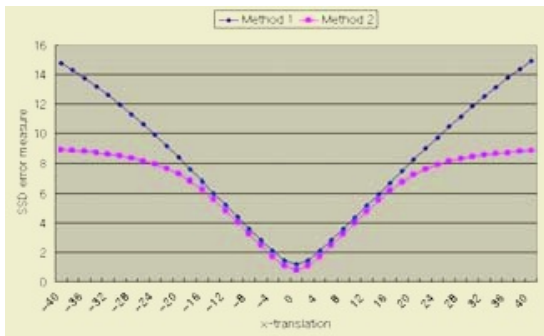

(a)

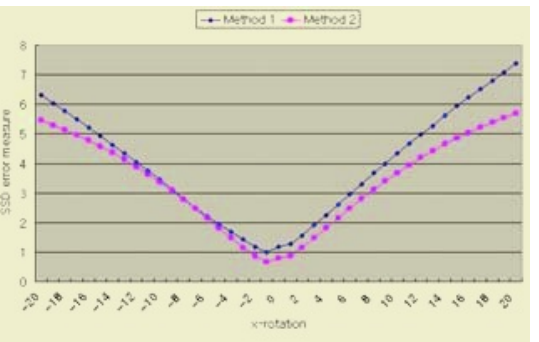

(b)

Fig. 9. Comparison of our proposed method and chamfer distance-based surface registration (a) the error in $\mathrm{x}$-translation (b) the error in $\mathrm{x}$-rotation

\section{Acknowledgements}

This work was supported in part by the Korea Research Foundation under the Brain Korea 21 Project. The ICT at Seoul National University provides research facilities for this study.

\section{References}

1. Yankelevitz, D.F., Reeves, A.P., Kostis, W.J., Binsheng, Z., henschke, C.I., Small Pulmonary Nodules: Volumetrically Determined Growth Rates Based on CT Evaluation, Radiology, Vol. 217 (2000) 251-256.

2. Betke, M., Hong, H., Ko, J.P., Automatic 3D Registration of Lung Surfaces in Computed Tomography Scans, Proc. Of Medical Image Computing and Computer-Assisted Intervention (MICCAI) (2001) 725-733.

3. Betke, M., Hong, H., Ko, J.P., Automatic 3D Registration of Lung Surfaces in Computed Tomography Scans, CS Technical Report 2001-004, Boston University.

4. Betke, M., Hong, H., Thomas, D., Prince, C., Ko, J.P., Landmark Detection in the Chest and Registration of Lung Surfaces with an Application to Nodule Registration, Medical Image Analysis, Vol. 7 (2004) 265-281.

5. Hong, H., Betke, M., Teng, S., Multilevel 3D Registration of Lung Surfaces in Computed Tomography Scans - Preliminary Experience, Proc. Of International Conference on Diagnostic Imaging and Analysis (ICDIA) (2002) 90-95.

6. Mullaly, W., Betke, M., Hong, H., Wang, J., Mann, K., Ko, J.P., Multi-criterion 3D Segmentation and Registration of Pulmonary Nodules on CT: a Preliminary Investigation.

7. Gurcan, M.N., Hardie, R.C., Rogers, S.K., Dozer, D.E., Allen, B.H., Hoffmeister, J.W., Automated Global Matching of Temporal Thoracic Helical CT Studies: Feasibility Study, Proc. of International Congress Series, Vol. 1256 (2003) 1031-1036. 